\title{
Programa de reabilitação funcional para sujeitos com sequelas de hanseníase*
}

\section{Functional rehabilitation program for subjects with leprosy sequelae}

\author{
Thiago da Silva Dias ${ }^{1}$, Jorge Lopes Rodrigues Junior ${ }^{2}$ \\ http://dx.doi.org/10.11606/issn.2238-6149.v27i3p355-360
}

Dias TS, Rodrigues Junior JL. Programa de reabilitação funcional para sujeitos com sequelas de hanseníase. Rev Ter Ocup Univ São Paulo. 2016 set.-dez.;27(3):355-60.

RESUMO: Este estudo objetiva analisar um programa de reabilitação funcional para sujeitos com sequelas de hanseníase. $\mathrm{O}$ estudo tem abordagem qualitativa e abrange a base teórica subjacente à aplicação do programa de reabilitação funcional a um caso típico de paciente com sequelas de hanseníase. $\mathrm{O}$ programa engloba técnicas de facilitação neuromuscular proprioceptiva (FNP), cinesioatividade e tecnologia assistiva (TA). Dentre os benefícios do programa, destaca-se que a FNP e a cinesioatividade podem viabilizar fortalecimento muscular, ganho de amplitude de movimento (ADM), resistência muscular e prevenção de deformidades, bem como os dispositivos de TA podem auxiliar no ganho de ADM, prevenção de deformidades e no desempenho das atividades cotidianas. Portanto, o programa terapêutico proposto neste estudo ressalta os benefícios da utilização integrada dos métodos e técnicas para a minimização de déficits funcionais (ADM, força e destreza manual) e ocupacionais em sujeitos com sequelas de hanseníase.

DESCRITORES: Atividades cotidianas; Hanseníase/ reabilitação; Equipamento de autoajuda; Terapia ocupacional.
Dias TS, Rodrigues Junior JL. Functional rehabilitation program for subjects with leprosy sequelae. Rev Ter Ocup Univ São Paulo. 2016 Sept.-Dec.;27(3):355-60.

ABSTRACT: This study aims to analyze a functional rehabilitation program for subjects with leprosy sequelae. The study has a qualitative approach and comprises the theoretical basis underlying the application of the functional rehabilitation program to a typical case of patient with leprosy sequelae. The program encompasses proprioceptive neuromuscular facilitation (PNF) techniques, kinesioactivity and assistive technology (AT). Regarding the benefits of this intervention, we highlight that PNF and kinesioactivity can enable muscle strengthening, gains in range of motion (ROM), muscle endurance, as well as prevention of deformities. In addition, the AT devices can enable gains in ROM, prevention of deformities, and improvements in the performance of daily activities. Therefore, the therapeutic program described in this study highlights the benefits of the integrated use of the methods and techniques above mentioned to minimize the functional (ROM, strength, and manual endurance) and occupational deficits for subjects with leprosy sequelae.

KEYWORDS: Everyday activities; Leprosy/rehabilitation; Self-help devices; Occupational therapy.

\footnotetext{
* Um resumo deste trabalho foi apresentado, em modalidade de pôster comentado, na X Semana da Saúde CCBS/UEPA - Saúde e Educação na Amazônia: desafios para o fortalecimento da rede de atenção à saúde, a qual ocorreu no período de 27 a 30 de Novembro de 2013.

1 Terapeuta Ocupacional. Discente de Mestrado no Programa de Pós-Graduação em Teoria e Pesquisa do Comportamento da Universidade Federal do Pará (UFPA), Belém, PA, Brasil.

2. Mestre em Doenças Tropicais (UFPA). Especialista em Reabilitação em Neurologia (UEPA). Docente do Curso de Terapia Ocupacional da Universidade do Estado do Pará - UEPA, Belém, PA, Brasil.

Endereço para correspondência: Thiago da Silva Dias. Programa de Pós-graduação em Teoria e Pesquisa do Comportamento, Universidade Federal do Pará - UFPA. Rua 8 de Maio, 252, Campina de Icoaraci, CEP 66813-110, Belém, PA, Brasil. Email: thiagodias.to@gmail.com.
} 


\section{INTRODUÇÃO}

A hanseníase é uma doença crônica granulomatosa, proveniente de infecção causada pelo Mycobacterium leprae, um bacilo com alta infectividade e baixa patogenicidade ${ }^{1}$. De fato, esta doença é caracterizada tanto por esta infecção bacteriana, que acarreta em intensa resposta imunocelular, como por uma neuropatia periférica iniciada com o quadro infeccioso e seus efeitos imunológicos².

Atualmente, apesar da melhoria na qualidade de vida e do conhecimento científico, esta doença continua tendo grande prevalência global. Em 2012 foram detectados 232.857 novos casos no mundo, sendo 36.178 pertencentes ao continente americano e, mais especificamente, 33.303 no Brasil ${ }^{3}$.

Conforme a classificação de Ridley \& Jopling, a doença varia entre dois polos - tuberculoide e virchowiano - e existem três formas intermediárias (grupo dimorfo). A forma dimorfa-virchowiana se caracteriza por lesões numerosas, pouco simétricas e com áreas anestésicas ${ }^{2,4}$.

A doença pode causar sérios prejuízos de caráter físico, social e/ou psicológico na vida do sujeito afetado². Porém, isto depende de fatores como: as características intrínsecas da doença, a relação com o hospedeiro, o grau de endemicidade do meio, entre outros aspectos ${ }^{1}$.

Quando não tratada precocemente, a hanseníase pode evoluir com diferentes tipos e graus de incapacidades físicas ${ }^{5}$. Cerca de $20 \%$ dos pacientes acometidos pela enfermidade evoluem com incapacidade física, necessitando de alguma intervenção para reabilitação ${ }^{6}$.

Portanto, de um modo geral, a doença tem alto potencial incapacitante. Entretanto, a melhoria das condições de vida e o avanço do conhecimento científico modificaram significativamente o quadro clínico da hanseníase, a qual, atualmente, tem tratamento e cura ${ }^{1}$.

Neste contexto, a Terapia Ocupacional utiliza diversas estratégias de intervenção voltadas para a prevenção de incapacidades e melhora do desempenho funcional e ocupacional. Desse modo, o tratamento foca na funcionalidade e no cotidiano da pessoa, de forma a possibilitar autonomia e/ou independência nas atividades diárias, incluindo autocuidado, trabalho, lazer e vida social ${ }^{7,8}$. Portanto, ressalta-se a priorização da prevenção de deformidades, preparação para a função e promoção da independência ${ }^{9}$. A intervenção abrange tanto a reabilitação da mão como a prevenção de incapacidades nos membros inferiores e face, locais frequentemente acometidos na hanseníase ${ }^{8}$. Isto é operacionalizado por meio de exercícios, técnicas de manipulação corporal, equipamentos específicos de mobilização, imobilização e/ou adaptação, entre outros procedimentos de tratamento 9 .

A prevenção de deformidades, através do uso de órteses, controle de edema e exercícios de movimentação passiva e ativa, é tão essencial quanto a restauração funcional, pois a instalação de uma contratura pode implicar em perda funcional com repercussões na independência do individuo".

Nesta perspectiva, um programa terapêutico ocupacional deve englobar métodos, recursos e técnicas que, em conjunto, viabilizem manutenção e/ou recuperação de funções necessárias para o desempenho funcional ocupacional dos sujeitos com sequelas de hanseníase. Dentre as várias possibilidades, este estudo destaca o potencial de um programa composto de facilitação neuromuscular proprioceptiva, cinesioatividade e tecnologia assistiva.

A Facilitação Neuromuscular proprioceptiva (FNP) é uma técnica de alongamento ativo que combina mecanismos fisiológicos do controle neuromuscular com a ativação dos impulsos dos órgãos tendinosos de Golgi ${ }^{10}$.

$\mathrm{O}$ termo cinesioatividade se refere a um recurso terapêutico ocupacional que associa técnicas cinesioterápicas à relação sujeito-objeto, de modo que as relações do corpo com o meio buscam conquistar a capacidade de executar movimentos hábeis ${ }^{11}$.

A Tecnologia Assistiva (TA) é uma área interdisciplinar de conhecimento, que engloba produtos, recursos, metodologias, estratégias, práticas e serviços que visam à promoção de funcionalidade, autonomia, independência, qualidade de vida e inclusão social de pessoas com deficiência, incapacidade e/ou mobilidade reduzida ${ }^{12}$.

Este estudo objetiva analisar o potencial de um programa de reabilitação funcional para sujeitos com hanseníase. Especificamente, objetiva-se indicar os benefícios da utilização integrada de facilitação neuromuscular proprioceptiva, cinesioatividade $\mathrm{e}$ tecnologia assistiva.

\section{PROCEDIMENTOS METODOLÓGICOS}

O estudo tem abordagem qualitativa e consiste em um relato de experiência baseado em estágio supervisionado na Unidade de Ensino e Assistência em Fisioterapia e Terapia Ocupacional (UEAFTO) e no laboratório de 
Tecnologia Assistiva (LABTA) da Universidade do Estado do Pará (UEPA).

Um caso típico é utilizado para destacar os déficits funcionais e o impacto da doença na vida cotidiana da pessoa com sequelas de hanseníase. Além disso, este caso demonstra a aplicação de um programa de reabilitação funcional desenvolvido durante o estágio supervisionado, o qual engloba técnicas de FNP, cinesioatividade e recursos de TA. Ressalta-se que a atuação do terapeuta ocupacional na UEAFTO e no LABTA é muito mais complexa e multifacetada.

O caso típico é um sujeito diagnosticado com Hanseníase Dimorfa-Virchowiana, o qual apresenta déficits de força, resistência e amplitude de movimento, bem como dificuldade na realização dos movimentos de preensão de força, pinça de precisão e dorsiflexão. Estes déficits influenciam em atividades cotidianas como alimentação, pentear o cabelo e escovar os dentes.

O arcabouço teórico subjacente ao programa de reabilitação funcional, incluindo aspectos clínicos da hanseníase e os princípios da atuação terapêutica ocupacional com esse público, advém de pesquisa exploratória realizada na Biblioteca da UEPA e em revistas científicas.

\section{RESULTADOS E DISCUSSÃO}

O plano terapêutico para o caso típico apresentado inclui os seguintes objetivos: prevenir deformidades e contraturas; promover ganhos motores em amplitude de movimento, força e resistência; melhorar a realização de preensão de força e pinça de precisão; e melhorar o desempenho de atividades de vida diária.

Neste contexto, destaca-se a seguir o potencial de cada componente do programa de reabilitação funcional para atingir os objetivos supracitados. Posteriormente, serão abordados os benefícios da utilização integrada destes componentes, ou seja, os benefícios do programa para sujeitos com sequelas de hanseníase.

\section{Facilitação neuromuscular proprioceptiva}

A FNP parece ser um método mais eficaz para ganhar alongamento muscular e amplitude de movimento (ADM) em pacientes com sequelas de hanseníase, em comparação ao alongamento estático passivo ${ }^{10}$.

Para o caso apresentado, pode-se utilizar, especificamente, a técnica de contrair-relaxar, visando ao aumento da flexibilidade das articulações do tornozelo
(AT), interfalangeanas (IFs) e metacarpofalangeanas (MTs).

O aumento da flexibilidade na técnica de contraçãorelaxamento ocorre por meio da contração muscular ativa, com o objetivo de ocasionar inibição autogênica do músculo alongado. Quando a técnica é aplicada, ocorre relaxamento muscular reflexo do músculo alvo, o que, associado ao alongamento passivo, promove o ganho de $\mathrm{ADM}^{10}$.

A contração concêntrica do músculo antagonista ao que está sendo alongado possibilita um alongamento maior, pois, através do principio de inibição recíproca, a contração muscular causa um relaxamento reflexo do músculo agonista, possibilitando um alongamento mais eficaz ${ }^{13}$.

Desse modo, esta técnica apresenta benefícios quanto à prevenção de encurtamento musculotendíneo, viabilização de ganhos em ADM, melhora na realização dos movimentos de pinça, preensão e dorsiflexão, assim como favorecimento do desempenho nas outras atividades realizadas durante os atendimentos.

\section{Cinesioatividade}

este recurso é utilizado com o objetivo de promover ganhos funcionais a partir do envolvimento do paciente em atividades que estimulem áreas lesadas e/ou com diminuição de função. O procedimento envolve a execução de movimentos vinculados a um referencial de ação, um objeto e/ou à execução de uma tarefa, que está revestida de um maior contingente de estimulação dado o seu contexto aplicativo, prático e visual ${ }^{11}$.

Nesta perspectiva, as seguintes atividades podem ser utilizadas para favorecer a execução dos movimentos de preensão de força, pinça de precisão e dorsiflexão: preensão e manipulação manual de bolas de tamanhos variados; manipulação de pinos de tamanhos graduados utilizando pinça de precisão; e manipulação de bolas de tamanhos variados utilizando os pés.

O plano de ação da cinesioatividade implica na promoção de várias tarefas, cada uma com um determinado número de repetições, de modo que, durante o processo, são desenvolvidas habilidades motoras básicas, as quais são, posteriormente, transpostas pelo sujeito para habilidadesalvo, ou seja, o movimento real envolvido nas atividades cotidianas $^{14}$.

O engajamento do sujeito em diversas atividades com os recursos supracitados objetiva viabilizar: ganho de ADM em MT, IFs e MTs, em associação ao alongamento ativo; aumento de força através do exercício promovido pela atividade; e melhora na realização dos movimentos 
de dorsiflexão, preensão de força e pinça de precisão, por meio da repetição regular das tarefas.

Este método viabiliza o desenvolvimento de habilidades motoras básicas, ou seja, a melhora em componentes de desempenho através de cinesioatividade. Posteriormente, isto pode influenciar o desempenho ocupacional, visto que as habilidades básicas são transferidas para movimentações funcionais constituintes de atividades realizadas cotidianamente pelo sujeito.

Neste sentido, destaca-se que a intervenção terapêutica ocupacional pautada na cinesioatividade facilita a recuperação e reeducação motora de indivíduos com disfunções que prejudiquem a sua funcionalidade ${ }^{15}$.

\section{Tecnologia assistiva}

A TA auxilia as pessoas com incapacidades físicas a realizarem suas tarefas através do uso de recursos tecnológicos ${ }^{16}$. Dentre estes recursos, destaca-se a utilização de adaptações funcionais e órteses no caso típico apresentado neste estudo.

As adaptações funcionais são recursos que viabilizam a recuperação de uma função perdida e estimulam a independência ${ }^{17}$. A prescrição de uma adaptação visa simplificar as capacidades funcionais e fornecer ao cliente a aquisição de domínio de si e do meio, bem como viabilizar uma sintonia entre cliente, tarefa e ambiente ${ }^{18}$.

Deste modo, considerando-se que o sujeito do caso supracitado apresenta déficits em atividades cotidianas específicas, ressalta-se a utilização de dois dispositivos frequentemente confeccionados no LABTA: adaptação para alimentação e adaptação para escrita.

A adaptação para alimentação consiste no aumento da área de preensão do cabo de talheres, utilizando-se Etil Vinil Acetato (EVA) e Velcro, a fim de facilitar a sua manipulação por indivíduos com déficit de força e ADM. Porém, ressalta-se que pode ser utilizada em pentes, escovas, e outros objetos a cujo cabo possa ser acoplado o dispositivo.

A adaptação para escrita consiste na simplificação da tarefa de preensão do lápis/caneta por meio da utilização de uma tira de couro para estabilização do objeto entre o polegar e o indicador, aos quais são acoplados tiras de Velcro.

Ressalta-se que as adaptações para Atividades de Vida Diária (AVDs) são especialidade do terapeuta ocupacional que atua em disfunções físicas e sensoriais, objetivando favorecer a funcionalidade de indivíduos que apresentam disfunções de origem ocupacional ${ }^{19}$.
Quanto às órteses, estes são dispositivos aplicados externamente ao corpo, a fim de modificar as características estruturais ou funcionais do sistema neuromusculoesquelético. Há, basicamente, quatro tipos de órtese: estática (imobiliza, alinha, posiciona e protege o tecido); dinâmica (aplica força para mover uma articulação); estática seriada e estática progressiva (mantêm o tecido em seu comprimento máximo para obter ganho de movimento ${ }^{20}$.

Neste contexto, destaca-se a utilização de um splint dinâmico de dedos para favorecer a realização de preensão de força. O dispositivo consiste em um componente de couro fixado no punho e cinco componentes, também confeccionados em couro, fixados em cada dedo, os quais são interligados por tiras elásticas que viabilizam a aplicação de força sobre as articulações metacarpofalangeanas.

Este pode ser utilizado durante as sessões, enquanto são realizadas as tarefas de cinesioatividade, a fim de atribuir maior nível de exigência motora às atividades realizadas e, assim, favorecer a prevenção de deformidades e encurtamentos, fortalecimento muscular, ganho de ADM e melhora na realização dos movimentos. Além disso, o paciente pode ser orientado a utilizar o dispositivo em casa, durante a realização das atividades cotidianas.

Quanto déficit na realização de dorsiflexão, destacase a utilização de uma órtese dinâmica do tipo Férula de Harris. Este dispositivo consiste em um componente confeccionado em couro, fixado na perna com tiras de Velcro, do qual parte uma tira elástica, fixada no calçado do individuo, a qual aplica uma força compensatória ao déficit de dorsiflexão.

A órtese, no geral, aplica um estresse externo à articulação de forma suave, a fim de controlar, preservar, modificar e influenciar a mobilidade, bem como obter resultados o mais breve possível. Aquelas do tipo dinâmica, especificamente, visam ao aumento da mobilidade articular nos casos de retração tendínea, processo cicatricial, contraturas e diminuição da ADM9 .

Ressalta-se que os dispositivos apresentados podem ser confeccionados com material de baixo custo no LABTA, o qual é utilizado pelos discentes do Estágio Supervisionado do $4^{\circ}$ Ano do Curso de Terapia Ocupacional da UEPA.

Neste sentido, destaca-se que os dispositivos de tecnologia assisitiva têm sido cada vez mais utilizados na reabilitação. Porém podem ter um alto custo financeiro e serem restritos a uma pequena parcela da população, por isso, no Brasil, utilizam-se diversos tipos de materiais alternativos e com menor custo ${ }^{21}$.

\section{Benefícios}


Os potenciais benefícios do programa de reabilitação funcional abrangem ganhos em ADM, força e resistência manual, verificadas por meio da progressão do paciente nas atividades relacionadas à realização de preensão de força, pinça de precisão e dorsiflexão durante os atendimentos. Além disso, pode-se prevenir deformidades e contraturas através da utilização do alongamento ativo e das órteses.

Por meio do relato do paciente e/ou da utilização da Medida Canadense de Desempenho Ocupacional (COPM), pode-se analisar as possíveis melhoras na realização de atividades cotidianas como alimentação, pentear o cabelo e escovar os dentes de forma mais independente, decorrentes tanto do alongamento ativo e da cinesioatividade como do uso dos dispositivos de TA.

Portanto, a utilização integrada dos métodos e técnicas supracitados pode alcançar os objetivos traçados para o caso típico apresentado, visto que a FNP e a cinesioatividade podem viabilizar fortalecimento muscular, ganho de ADM, resistência muscular e prevenção de deformidades, bem como os dispositivos de tecnologia assistiva podem auxiliar no ganho de ADM, prevenção de deformidades e no desempenho das atividades cotidianas.

\section{REFERÊNCIAS}

1. Brasil. Secretaria de Vigilância em Saúde. Guia de vigilância epidemiológica. 7a ed. Brasília: Ministério da Saúde; 2009.

2. Scollard DM, Adams LB, Gillis TP, Krahenbuhl JL, Truman RW, Williams DL. The continuing challenges of leprosy. Clin Microbiol Rev. 2006 Abr;19(2):338-81. doi:10.1128/ CMR.19.2.338-381.2006.

3. World Health Organization. Global leprosy: update on the 2012 situation. Wkly Epidemiol Rec [Internet]. 2013 Ago 30 [Citado 01 Fev 2016]; 35(88):365-80. Disponível em: http://www.who.int/wer/2013/wer8835.pdf?ua=1.

4. Rodrigues LC, Lockwood DN. Leprosy now: epidemiology, progress, challenges, and research gaps. Lancet Infect Dis. 2011 Jun;11(6):464-70. doi: 10.1016/S1473-3099(11)70006-8.

5. Alves CJ, Barreto JA, Fogagnolo L, Contin AL, Nassif PW. Avaliação do grau de incapacidade dos pacientes com diagnóstico de hanseníase em serviço de dermatologia do estado de São Paulo. Rev Soc Bras Med Trop Dis. 2010;43(4):460-1. doi:10.1590/S003786822010000400025.

\section{CONCLUSÕES}

A utilização integrada da facilitação neuromuscular proprioceptiva, cinesioatividade e tecnologia assistiva pode trazer benefícios para a minimização de déficits funcionais (ADM, força e destreza manual) e ocupacionais (alimentação, pentear cabelo, escovar os dentes). Desse modo, estes métodos e técnicas podem ser utilizados em um programa de reabilitação funcional para pessoas com sequelas de hanseníase.

Ressalta-se que a atuação do terapeuta ocupacional na UEAFTO é multifacetada e complexa. Desse modo, o programa terapêutico apresentado neste estudo não abrange todo o escopo de atuação terapêutica ocupacional, mas representa uma proposta de intervenção com base em déficits funcionais e ocupacionais que são comumente identificados em indivíduos com sequelas de hanseníase.

O estudo apresenta como limitação o caráter teórico e o número reduzido de pesquisas englobando, principalmente cinesioatividade e facilitação neuromuscular proprioceptiva na área de Terapia Ocupacional. Por isso, este estudo visa suscitar a realização de pesquisas empíricas abrangendo um ou mais dos métodos e técnicas componentes do programa terapêutico.

6. Oliveira DT. Avaliação de sequelas neurológicas em pacientes com hanseníase no estado de Sergipe [Dissertação]. Sergipe: Universidade Federal de Sergipe; 2012.

7. Peixoto AC, Martinho, NJ, Peixoto, AL, Pereira, GF, Landim, FL. A atuação da terapia ocupacional em uma antiga colônia de hanseníase no estado do Ceará. Cad Saude Colet [Internet]. 2008 Jan/Mar [Citado 01 Fev 2016];16(1):67-82. Disponível em: http:/www.cadernos. iesc.ufrj.br/cadernos/images/csc/2008_1/artigos/CSC_ IESC_2008_1_5.pdf.

8. Loureiro LA. Terapia ocupacional e hanseníase - reabilitação e seus significados [Dissertação]. Niterói: Universidade Federal Fluminense; 2012.

9. Gollegã AC, Luzo MC, De Carlo MM. Terapia ocupacional - princípios, recursos e perspectivas em reabilitação física. In: De Carlo, MM, Bartalotti CC, organizadores. Terapia ocupacional no brasil: fundamentos e perspectivas. São Paulo: Plexus Editora; 2001. p. 137-54.

10. Diaz AF, Moro FL, Binotto, JM, Fréz, AR. Estudo comparativo preliminar entre os alongamentos 
proprioceptivo e estático passivo em pacientes com sequelas de hanseníase. Fisioter Pesqui. 2008 Out/Dez;15(4):339-44. doi:10.1590/S1809-29502008000400004.

11. Vasconcelos M.H. Cinesioatividade: reorganização da memória de procedimento em hemiplégicos no tocante ao espaço de alcance e seu significado no cotidiano destes sujeitos [Dissertação]. Fortaleza: Universidade de Fortaleza; 2003.

12. Brasil. Subsecretaria Nacional de Promoção dos Direitos da Pessoa com Deficiência. Tecnologia assistiva. Brasília: CORDE; 2009.

13. Conceição AO, Dias GA. Alongamento Muscular: uma versão atualizada. Latu \& Sensu [Internet]. 2004 Jun [Citado 29 Jan 2016];5(1):136-41. Disponível em: http://pt.scribd. com/doc/52887007/Alongamento-Muscular\#scribd.

14. Vasconcelos M.H. Cinesioatividade: espaço de reeducação funcional para disfunção neuromotora em adultos. RBPS [Internet]. 2004 [Citado 27 Jan 2016];17(3):149153. Disponível em: http://www.redalyc.org/articulo. oa? $\mathrm{id}=40817308$.

15. Beckman KA, Santos NC. Terapia ocupacional: relato de caso com vítima de escalpelamento por eixo de motor de barco. Cad Ter Ocup UFSCar [Internet]. 2004 [Citado
27 Jan 2016];12(1):20-43. Disponível em: http://www. cadernosdeterapiaocupacional.ufscar.br/index.php/ cadernos/article/view/189/145.

16. Rodrigues AC. Reabilitação: práticas inclusivas e estratégias para a ação. São Paulo: Andreoli; 2008.

17. Alves de Oliveira, AI, Lourenço JM, Garotti MF. Tecnologia assistiva: pesquisa e prática. Belém: EDUEPA; 2008.

18. Neistadt ME, Crepeau EB. Willard \& Spackman Terapia Ocupacional. 9a. ed. Rio de Janeiro: Guanabara Koogan; 2002.

19. Cruz DM, Meneses KV. Prescrição de Adaptações. In: Cruz DM, organizador. Terapia ocupacional na reabilitação pósacidente vascular encefálico - atividades de vida diária e interdisciplinaridade. São Paulo: Santos; 2012. p. 103-16.

20. Assumpção TS. Órteses - princípios básicos. In: Freitas PP, organizador. Reabilitação da mão. São Paulo: Editora Atheneu; 2006. p.539-53.

21. Klein AN. Uso da tecnologia para pacientes com esclerose lateral amiotrófica. Revista Neurociências [Internet]. 2006 Abr/Jun [Citado 02 Fev 2016].14(2 suppl):70-1. Disponível em: http:/www.revistaneurociencias.com.br/edicoes/2006/ RN\%2014\%20SUPLEMENTO/Pages $\% 20$ from $\% 20$ RN\%2014\%20SUPLEMENTO-10.pdf.

Recebido em: 02.02.16

Aceito em: 06.09.16 